\title{
A Blockchain Approach Applied to a Teledermatology Platform in the Sardinian Region (Italy) ${ }^{\dagger}$
}

\author{
Katiuscia Mannaro, Gavina Baralla *, Andrea Pinna and Simona Ibba \\ Department of Electric and Electronic Engineering, University of Cagliari, Cagliari 09123, Italy; \\ mannaro@diee.unica.it (K.M.); a.pinna@diee.unica.it (A.P.); simona.ibba@diee.unica.it (S.I.) \\ * Correspondence: gavina.baralla@diee.unica.it; Tel.: +39-070-675-5890 \\ + This paper is an extended version of our paper published in The fifth international IEEE workshop on \\ e-Health Pervasive Wireless Applications and Services (e-HPWAS'17).
}

Received: 15 January 2018; Accepted: 19 February 2018; Published: 23 February 2018

\begin{abstract}
The use of teledermatology in primary care has been shown to be reliable, offering the possibility of improving access to dermatological care by using telecommunication technologies to connect several medical centers and enable the exchange of information about skin conditions over long distances. This paper describes the main points of a teledermatology project that we have implemented to promote and facilitate the diagnosis of skin diseases and improve the quality of care for rural and remote areas. Moreover, we present a blockchain-based approach which aims to add new functionalities to an innovative teledermatology platform which we developed and tested in the Sardinian Region (Italy). These functionalities include giving the patient complete access to his/her medical records while maintaining security. Finally, the advantages that this new decentralized system can provide for patients and specialists are presented.
\end{abstract}

Keywords: teledermatology; blockchain; smart contracts; e-health

\section{Introduction}

Telemedicine is a form of technology which is particularly suited to the medical specialty of dermatology. Teledermatology is, as such, one of the more common applications of e-health. Teledermatology in primary care has been shown to be reliable, offering the possibility of improving access to dermatological care, for example through use of telecommunication technology to connect several medical centers over long distances, enabling the transfer of information about skin conditions.

Some of the aims of the teledermatology include ensuring that patients from remote areas receive specialized dermatological care, which thus decreases the number of hospital visits and increases diagnostic efficiency [1]. Thus, high quality health care is delivered more efficiently by moving the patient's information rather than moving the patients themselves [2]. In past decades, specific teledermatology programs allowed the significant diffusion of teledermatology services. At the beginning of 2012, 37 teledermatology programs were active in the U.S. The dominant delivery modality was the store-and-forward technique. The sustainability of teledermatology programs depends on financial programs and on health legislation [3]. Our project starts with the assumption that e-health may be improved with respect to security and healthcare data management thanks to blockchain technology. In the last few years, there has been increased interest in blockchain technology as a new model of data sharing in several domains such as medical research or in smart city contexts. Blockchain technology is an innately secure way to share data, using automatic cryptographic checking of data consistency. In particular, data is stored in a digital ledger composed of a linked block of transactions. A blockchain is usually a decentralized and peer-to-peer based system.

Recently, different blockchain-based telemedicine projects have been launched. These are mostly initial coin offering (ICO) initiatives. ICOs are mainly used as fundraising for projects 
built on blockchain technology. We cite below some examples of telemedicine ICOs. The DocCoin (https://www.doc-coin.com/) service enables patients to receive counseling, referrals for tests, prescriptions for medicines, and diagnoses from doctors from all over the world. PointNurse (https://www.pointnurse.com) is a virtual care network platform for delivering direct primary care and care management services. By using "care credits", nurses, doctors, and other healthcare specialists can provide consultations to patients. LindaHealthCare (https:/ / linda.healthcare/) offers a medical assistant using artificial intelligence. The researchers state that they are developing conversational artificial intelligence and natural language processing for primary care, general health, and medicine. The MedicalChain (https://medicalchain.com) platform stores and shares health records with different groups such as doctors, hospitals, laboratories, and pharmacists, and health insurers can request permission to access medical records for their purposes. The OpenLongevity (http:/ / en.openlongevity.io/) platform consists of a website, a mobile app including a neural network to give advice on lifestyle, sports, and nutrition, and a telemedicine database to obtain a consultation based on personal data. The MedCredits (https://medcredits.io/) platform connects patients and doctors worldwide through a decentralized network. The company claims that the first software release will focus on the field of dermatology.

This paper presents some points of the implementation of a teledermatology project, known as DermoNet, in Sardinia, which is an insular Italian region. The DermoNet project (see [4] for more details) was financed by the Sardinia Region with the aim of developing a virtual organization model for teledermatology purposes by designing, developing, and validating a software platform based on cloud technology to create a network involving patients, general practitioners (GPs), dermatologists, and health care institutions, supporting clinicians during the evaluation of dermatological patients. Originally, the DermoNet project was implemented as a cloud-based information system with resources dynamically scaled and used over the Internet in order to improve health care services. DermoNet aims to be a useful tool to create a network for dermatological teleconsultation and provide dermatological support to general practitioners, particularly in underserved areas in the island of Sardinia. The offered consultation service allows the diagnosis and management of patients with skin diseases by GPs or dermatologists. In short, DermoNet enables each patient to contact his GP in reference to dermatological problems without physically needing to go to a specialist. This provides benefits in terms of time and costs. The system may provide answers about some serious dermatological diseases such as melanoma through a virtual organization (VO), a new form of organization made possible by the use of online services. In fact in VOs, doctors that work in distant places can access the same collaborative resources on the web and GPs can therefore take advantage of teleconsulting. In light of the new technologies, we have subsequently designed additional functionalities for the proposed system by using an alternative approach to develop a blockchain-based teledermatology platform. Through a decentralized system a patient can autonomously access his data and request a consultation from a specialist without general practitioner or hospital assistance.

Finally, the paper underlines the main differences and the advantages or disadvantages between the two different platforms by discussing limits and applications in different contexts such as rural areas where communication and health services still require development.

The paper is structured as follows: Section 1 provides an introduction of the paper. Section 2 presents the state of the art. Section 3 describes the DermoNet project and the methodology used. Section 4 describes an alternative blockchain based e-health platform. Section 5 discusses the advantages and disadvantages of the two different approaches, and finally, conclusions are discussed in Section 6.

\section{Related Work}

This section presents some works related to this project.

In recent years, increasing development of telemedicine applications has occurred, with the aim of satisfying patient needs regardless of age or location. 
Chen et al. [5], with respect to unbalanced healthcare services between rural and urban areas, analyzed the importance of usability indicators for a web portal in a tele-healthcare program. The authors collected data from urban and rural areas in Taiwan, and showed that urban patients tended to use the web portal more often than rural patients, providing more advanced information. This highlights the lack of services in rural areas, as well as the necessity to develop additional services such as telehealthcare.

In [6] authors describe IReHMo, a system which combines several IoT CoAP-based protocols [7], to remote monitor medical data and take care of elderly people at home. The system must manage a large amounts of data, and the authors discuss how to efficiently transmit healthcare data, especially from remote areas.

Given the strong development of context-aware technology and IoT devices designed for health monitoring, Hussain et al. [8] implemented a people-centric sensing system to provide real-time healthcare services for elderly and disabled people in cases of emergencies or abnormal health conditions.

According to Hossain [9] a smart home health care system could be useful for elderly people that can be constantly monitored. The described system uses a combination of tools such as microphones and video cameras installed in houses with the aim of constantly capturing images and patient speech for real-time monitoring. All data is stored in a cloud server.

Kroemer et al al. [10], in a skin tumor screening context, evaluated the diagnostic accuracy of clinical dermoscopic image tele-evaluation. Over a period of three months, they acquired images from 322 clinical cases and 278 dermoscopic cases using a mobile phone camera. The authors found that clinical image tele-evaluation might be the method of choice for mobile tumor screening.

Rosado et al. [11] state that "mobile teledermatology is a promising tool with the potential to empower patients to adopt an active role in managing their own health status." In their work, they present a mobile-based framework developed to provide an early diagnosis of skin cancers. The described system is composed of a mobile application for patients, a server to process and store images, and a web interface for dermatologists.

According to Kanthraj [12], patient-assisted teledermatology practice is a new model developed in the health-care context. In this model, a patient can upload his medical data, (i.e., clinical images) as a first point of contact to request a consultation or a face-to-face examination followed by a teledermatology consultation.

Finally, Jaworek et al. [13] proposed a cloud-based teledermatology system for the fast and efficient exchange of information. The system supports the consultation process between general practitioners and dermatologists, and it can be useful in case of inexperienced doctors when the application is used as a decision support system.

In contrast, the use of blockchain technology in the medical field is a very recent development. In [14] the authors face the problem of health information traceability using tokenization. In this system, all patients can be paid for sharing their own health data. A token is used as incentive to involve more people in the blockchain-based application. The blockchain technology is useful not only for ensuring good digital healthcare, but also for managing problems of interoperability and privacy with the opportunity for patients to be the focus of all of their personal health data.

According to Petrenko et al. [15] blockchain technology represents a viable solution to one of the most important requirements of health informatics systems: data security. They assume that patient's information has to be encrypted by a trusted cryptographic algorithm, stored, and shared on blockchain in order to provide security and avoid problems of unavailability. The most important feature of this solution is the invariability of data recorded on the blockchain. In addition, it allows patients to provide the terms of access to their own information whilst fully respecting privacy rules, while allowing all interested parties to use anonymous data for research activities.

In contrast, ProActive Aging [16] is a platform that aims to support the active living of aging people. It is blockchain-based and provides a solution to manage the identities of patients. In [17] 
Shubbar presents a telecommunication healthcare infrastructure for remote monitoring of the treatment of cancerous tumors. It uses blockchain technology and smart contracts. The platform is used in specialized medical centers as well in patient homes. To classify images and calculate tumor size, a support vector machine (SVM) algorithm is used for image classification. Genestier at al. [18] propose a blockchain approach to manage access to personal data in the e-health area dealing with challenging security and privacy aspects. Yue et al. [19] deal with healthcare data management, proposing an App with blockchain-based architecture to enable patients to own, control, and share their data in a easy and secure way without privacy violations.

In this paper, we present the DermoNet project and in addition we also design an alternative blockchain-based e-health platform in which the patients can request a dermatological consultation, bypassing the GP and self-managing their own medical records.

\section{The DermoNet System}

In this section, we describe the primary DermoNet system. We studied a solution to provide dermatological services directly by the general practitioner with the purpose of virtually shortening the distances between patients and dermatologists. General practitioners provide preventive and routine health care to patients, playing a key role in the prevention, diagnosis, and monitoring of diseases. They are responsible for the initial assessment of skin lesions (10-15\% of primary care cases). Due to their widespread presence, GPs are a crucial element for ensuring high levels of health care, even in sparsely populated areas, where it is often expensive and difficult to get to a specialist. However, GPs need to see dermatology cases frequently enough to remain competent. Teledermatology services provide a diagnostically reliable method of increasing access to expertise in resource-poor regions.

Many studies [20-22] have highlighted the necessity of distributed medical services, leading to the design of a special typology of virtual organization (VO). We chose to use a VO because can offer solutions to many different dermatological problems in a timely manner. It in fact consists of multiple and distributed stakeholders temporarily linked together for a common process or value chain, supported by distributed information technology. The main characteristics of VOs [23] such as rapid community formation, the fast training of personnel involved, and the absence of long-term commitments, make this approach useful in medical circles in the case of emergencies, or for the rapid diagnosis of disease.

According to the definition of VOs provided by Tamosiunaite [24] "a VO is a self-sufficient geographically distributed short time or permanent consolidation of organizations, groups or individuals, which/who without time or space hindrances communicate through ICT (Information Communication Technology) while reaching a common goal." In the teledermatology context, a common goal of the VO participants is the increased quality of health services. The most important participants are general practitioners and dermatologists. The overall value of the virtual organization increases as new participants join it. The more GPs that join the VO, the greater the capillarization and spread of teledermatological services across the region. Dermatologists increase the value of the VO by means their expertise and their availability to be called for a teleconsultation. The VO resolves the problems of distance and insularity in regions such as Sardinia. The establishment of the VO requires an ICT infrastructure. We have focused on the creation of this infrastructure by means of the development of a web portal. The portal is a web-based system with which accredited users can operate using the system functionalities.

In detail, the DermoNet-integrated software system, in short the DermoNet System, is a pre-industrial prototype of a collaborative software platform of services oriented to support the management of dermatological patients and the diagnosis process by general practitioners and specialists working together in the VO.

Furthermore, the DermoNet System has been developed to ensure software interoperability. Indeed, DermoNet works with a heterogeneous set of systems that comprise the VO. Therefore, 
information contained in DermoNet is standardized and structured. It is made available by using tools designed to store and share data.

The software interoperability requirements, along with specific design requirements of dermatology functionality, highlight the importance of a well-defined knowledge base. In order to manage the information, such as document retrieval using keywords or meaning, the system is based on three different knowledge bases (KBs).

The software platform uses three KBs, and in runtime it allows a deduction process through a reasoner for the normal system operations. By using the API, a user can query each KB.

In order to manage information, such as document retrieval by using keywords or other attributes, the system is based on three different knowledge bases (KBs). We have named these KBs as follows:

1. $\mathrm{KB}_{G L}-\mathrm{A}$ knowledge base related to the guidelines for dermatologists. Guidelines contain clinical behavior recommendations with the aim of helping doctors to choose the most medically appropriate approach.

2. $\mathrm{KB}_{I}-\mathrm{A}$ knowledge base related to all images available within the dermatological atlas.

3. $\mathrm{KB}_{C E}-\mathrm{A}$ knowledge base related to the expertise of dermatologists that work in the competent centers and that provide teleconsulting services.

Thanks to the KBs, two important features are obtained, namely data shareability and the data linking. KBs represented the starting point in order to obtain an effective data management. Furthermore, by means of the KBs, the software system can provide a content-based search of documents and information that works taking in account the meaning of the search terms. The DermoNet portal is reached via a web client and works on the Internet. The portal is developed in order to be fully responsive and capable of working on mobile devices like tablets and smartphones.

\subsection{The DermoNet Portal}

Users access to the system through a login page designed to work with one-time password (OTP) system. The system main menu is composed of a list of available functionalities. The number of displayed functionalities depends on the user's typology (i.e., general practitioner, dermatologist specialist, tutor, secretary). A special account, providing access to the configuration pages, is enabled for the system administrator. The main menu summarizes the DermoNet functionalities. It contains the following entries.

Staff components-opens the team definition page. Teams can be created choosing from the list of DermoNet users, filtering by category, competence area and department. It represents the starting point to define the VO. The questionnaire model opens the questionnaire creation page. The GP can write personalized and interactive questionnaires, which on the basis of the patient's answers will show different question sequences.

Patients-opens the patient's management page. This page provides a form with which is possible to add and edit patient information. Only accredited users as the tutor or secretary have full access to the functionalities.

Medical Record - opens the medical record page. This page appears as shown in Figure 1. For each patient, the system displays an interactive form where the patient's name, general practitioner, and dermatology specialists are highlighted. The Medical Record page provides the list of treatment plans and the list of therapeutic annotations.

Appointments—opens the appointments book.

Reports-opens the report section with which it is possible to generate formatted views of the user data. 
Dermatological functionalities - opens the dermatological section in which all the system functionalities dedicated to the teledermatology are provided. These represent the core of the DermoNet system. The whole set of functionalities will be discussed in the following sub-section.

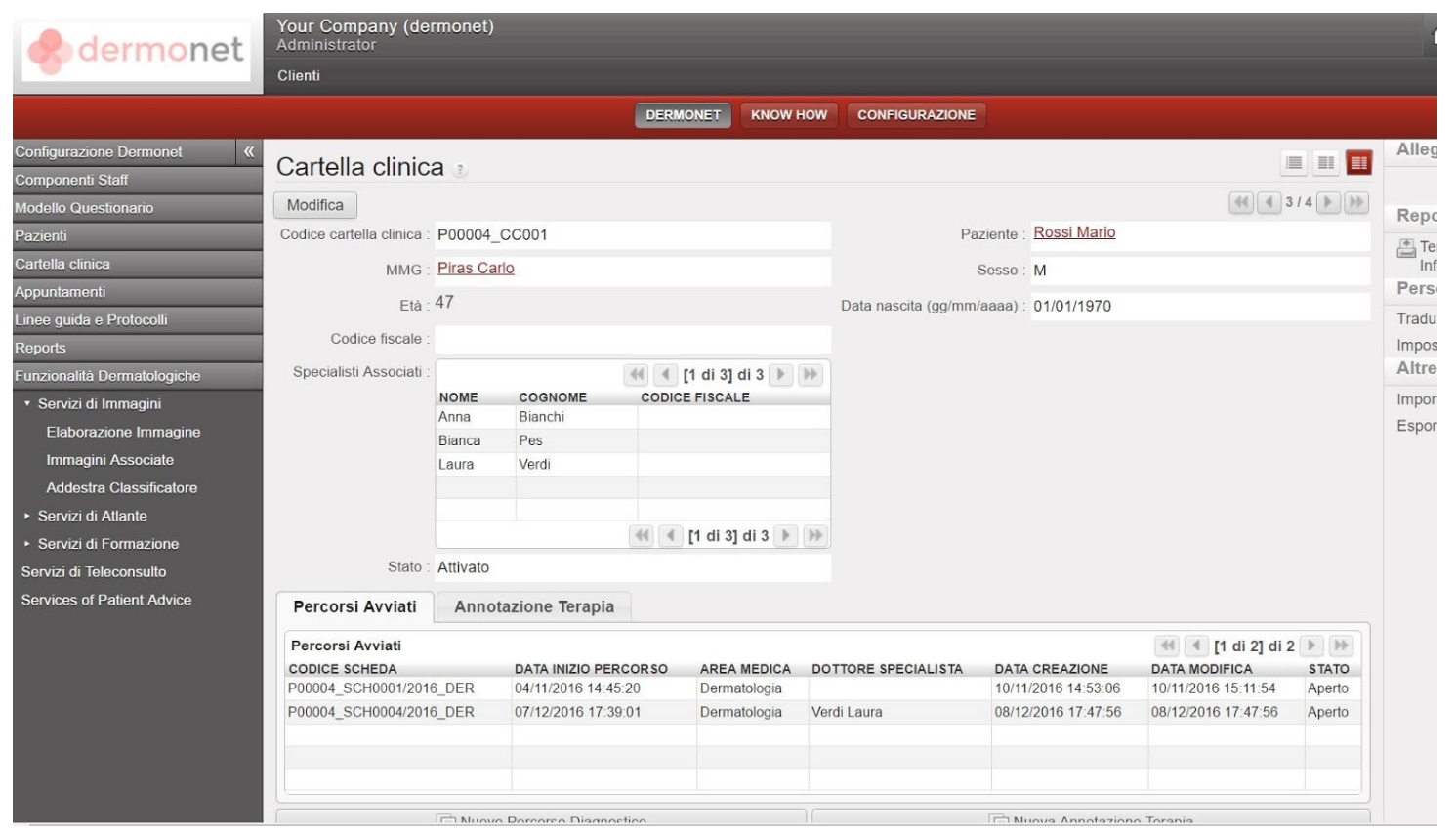

Figure 1. Patient medical record.

\subsection{DermoNet Dermatological Functionalities}

As mentioned above, the DermoNet system is based on the construction of specific KBs. Thanks to it, the system is able to provide advanced dermatological functionalities. The most relevant dermatological functionalities are described as follows.

Images analysis. The DermoNet prototype provides an automatic analysis and annotation system for dermatological images. The system provides a semantic annotation of classification and diagnosis probability on the basis of the KBI described above. Figure 2 shows a screenshot of some pictures of skin disorders.

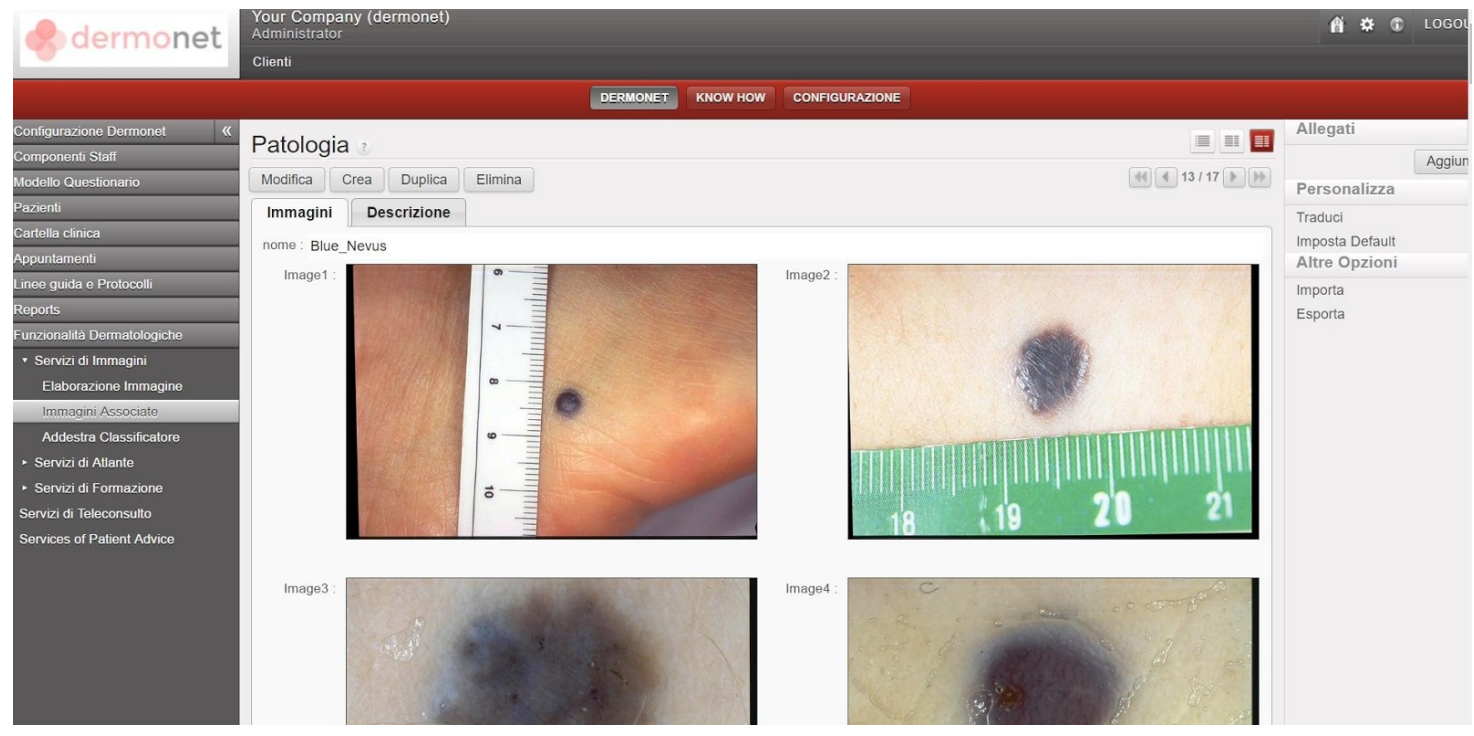

Figure 2. Pictures of skin disorders. 
Guideline services. These services enable dermatologists to consult and receive updates on diagnosis and therapy using the Guideline Knowledge Base. These guidelines contain clinical behavior recommendations and advice in order to help doctors choose the most medically appropriate assistance. The guideline services allow for different perspectives to be considered, for example points of view and needs of patients, organizational and ethical aspects, and of course the effectiveness of treatments relating to skin disorders.

Atlas service. The dermatological atlas provides services in order to catalog and share specialized dermatologic data. This service is based on the Image Knowledge Base. Users can explore the Atlas using a semantic search engine.

The semantic search engine works with the KBs described previously. It is possible look for information on a specific disease by providing the localization or the diagnosis, followed by the terminology recorded within the KBs. Results are grouped by diagnosis or by localization.

Teleconsulting services. DermoNet provides an environment for teleconsulting, which occurs between the GP and a specialist in the VO. Teleconsulting is facilitated by the Expertise Dermatology Competence Knowledge Base. It provides the data and terminology needed to avoid misunderstandings during the communication between GP and the specialist. Some other services provided by the DermoNet portal include learning services and patient advice solutions.

\subsection{Methodology}

In order to understand how the implementation of a virtual organization using teledermatology is able to improve the quality of care, and to put into effect smart growth strategies for specific regions such as the Sardinian island, a qualitative study was conducted. This study aimed to identify the perceptions of general practioners and dermatologists. General practitioners and dermatology specialists who participated in the project were invited to express a personal opinion on the elements of satisfaction or technical-professional dissatisfaction with respect to the DermoNet system in specific cases. For sake of brevity, we will describe here below only two particular use cases, as shown in Figure 3.

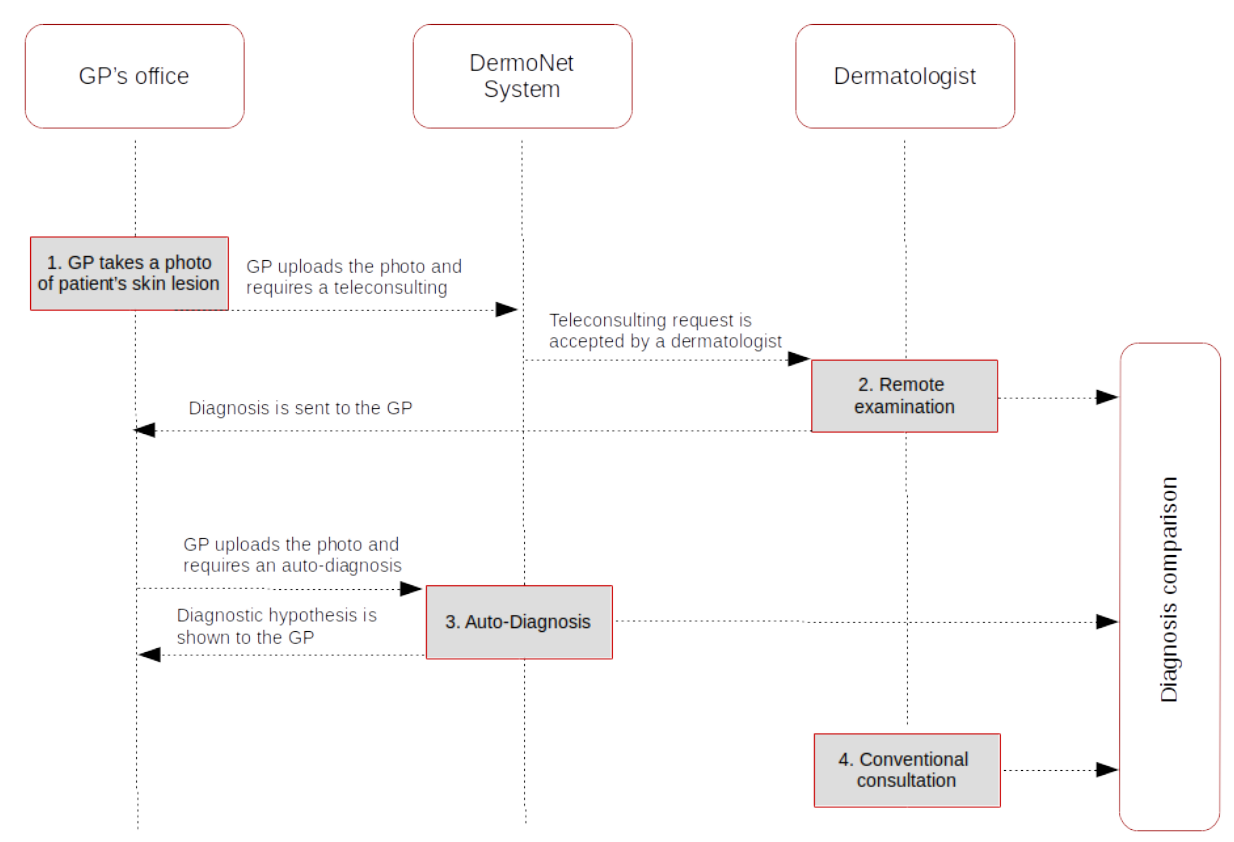

Figure 3. Diagnosis procedures: two use cases. GP: general practitioner.

In the first use case, teledermatology consultations were carried out in the offices of the GPs who participated in our project. Patients with skin lesions were informed about the project and signed 
an informed consent form. Then, GPs took photographs of lesions using a smart phone and filled in the consultation request forms available in the software system, attaching clinical images and providing some information. Finally, the request for the diagnosis was associated to a specific dermatologist. Then, the same patient was referred for a face-to-face consultation with the same dermatologist in order to compare the two diagnoses. Secondly, in this project an automatic classification method of skin lesions from dermoscopic images was proposed. We will not describe our adopted method of classification of skin lesions because this is not the purpose of the paper. In the second use case, we tested if the system was an effective tool for the diagnosis of skin lesions using dermoscopy images. The GP took photographs of the lesion and uploaded them in the system for automatic diagnosis of skin lesions. The algorithm is capable of segmenting skin lesions in dermoscopy images, classifying them for detecting melanomas, and separating them from other lesions. The system gives the GP a diagnosis without the consultation of a specialist. Finally, the same patient was referred for a face-to-face consultation with a dermatologist in order to compare the two diagnoses. We used the same classification of levels of agreement both for the dermatologists and the GPs. We measured the satisfaction level in terms of impact on the health system by following the smart region paradigm because the GPs and dermatologist specialists were located in different municipalities of the Sardinian region.

The number of interviews may be considered small (10 general practitioners and 3 dermatology specialists), but we followed the principle of saturation, in which data is collected until a saturation of information is obtained $[25,26]$. The analysis of collected data revealed a not entirely homogeneous framework. This was due to the reticence of some GPs towards the use of the technologies, and the fact that in general, obstacles to the spread of telemedicine are also of a technological nature (as confirmed by some studies).

All general practictioners declared the ability to share healthcare data with dermatologists as being very helpful. Considering this feedback, we formulated a specific question regarding this theme. All interviewees confirmed what their colleagues suggested, and hence the functionalities of access and information-sharing of the system DermoNet related to reporting, diagnosis, and photographs of the pathology are definitely useful for improving their work. Another important key point was related to ethical and regulatory aspects, including any legal-medical implications. These considerations are also derived because nowadays there are guidelines available on the matter, without specific regulations in this area. All the interviewees agreed that the prototype was perceived as a powerful tool to improve dermatological services for populations living in remote areas.

\section{The Blockchain E-health Platform}

In this section, we describe an alternative e-health platform, designed in order to use blockchain technology, that may be applied to the teledermatology domain. In the following, we first provide an overview of the blockchain technology and smart contracts. Then, we describe the architecture of the proposed platform.

\subsection{Blockchain Technology and Smart Contracts}

Blockchain technology is a data structure of the decentralized ledger technology (DLT) family that generates trust and ensures data integrity without the need of a trusted third part. A blockchain is shared in the nodes of a peer-to-peer network and each node stores a copy of it. Data recorded inside the blockchain is obtained through transactions. From a general and high-level point of view, a transaction is a valid message between two authorized accounts. In particular, each blockchain implementation includes validation algorithms, message protocols, and a data-upgrading strategy. Nowadays, the first and most important blockchain implementation is the bitcoin system [27]. This system introduced the blockchain as the solution to the "double spending" problem in digital money transfer.

In this blockchain, transaction data is organized in blocks. Each block is digitally signed (by means of a hashing operation) and linked to the previous one. This makes data immutable and defends 
integrity. Block creation is a task entrusted to network nodes that listen for transaction requests waiting to be validated. Transactions are composed of a list of sender accounts, a list of receiver accounts, and of an amount of digital coins (called bitcoins). The transaction is valid only if each sender wants to transfer coins that he/she has previously received (by means a transaction already recorded in the blockchain). In bitcoins, the block creation passes through a proof-of-work, a cryptographic puzzle for which the difficulty is proportional to the total computing power of the network. Those which first solve the cryptographic puzzle are called "miners" and obtain a prize in bitcoins, that can be spent with a transaction.

The second step in DLT evolution consists in the advent of the blockchain-based Decentralized Platform for Applications. In this case, the most important implementation is the Ethereum system [28]. Ethereum introduced a new typology of account, a robotic account that lives inside the blockchain. This type of account is known as a contract (or smart contract, as coined by Szabo [29] in 1997). Like normal accounts, a contract is able to both receive and send transactions. In addition, a contract contains a computer program that makes it able to compute and store data automatically.

In particular, smart contract programs contain a set of functions that other accounts can call by means of a specific transaction. Indeed, Ethereum transactions are messages that concern not only coin transfer.

The Ethereum blockchain is a state machine and each change in stored data (i.e., account balances, smart contract data, etc.) passes through a state transition. A new state depends on the data recorded in the previous state and on transaction bodies.

Because of the energy effort of network nodes, each blokchain operation has a cost. In the bitcoin system, users can pay a voluntary fee to the network for each transaction. In the Ethereum system, the fee mechanism revolves around the gas. In particular, the execution of each transaction and of each smart contract instruction requires a certain amount of gas. Operations have different gas costs. For example, storing data is more expensive than executing an arithmetic sum. Conversely, reading stored data is free. The gas price is not fixed. Users can decide how much pay it. However, users have to take in account that if the chosen gas price is too low, the network can ignore their requests.

Smart contracts purposes range from crowdfunding campaigns and money control to supply chain management and transparency [30]. They also allow the creation of decentralized organizations in which rules are written inside the code, in a programming language. Permission management, data availability, and validation and checking operations are typical features that smart contracts can provide through the decentralized platform.

\subsection{Platform Architecture and Use Cases}

In order to focus on the domain of the system, we first introduce the actors and their interactions. Then, we describe the architectural structure of the system. The e-health alternative platform for teledermatology is blockchain-based, and characterized by a decentralized organization that involves three types of actors. These are patients, specialists, and medical community members.

Patients are actors that need dermatological consultations, specialists make available their experience, and medical community members can access decentralized data for research activities such as clinical trials. To interact with the system a registration is required, and for each actor an identifier (such as a blockchain address) is created and recorded within the smart contracts. Unlike the DermoNet Virtual organization, in the decentralized system participants' operations are managed by the entire network system (accessing, communication, and authorizations) by means of the decentralized application running in the blockchain that makes the patient free to use the system independently.

We can have three different profiles: those of the patient, doctor, and medical community member. Each participant has a different role and type of access. Smart contracts manage all the operations and communications between actors and the platform depending on their profiles. All these operations are transparent to the user that logs on to the system by using a mobile App or a software application. 
A patient can ask for a dermatological consultation by sending a photo and all supporting data for a diagnosis. To send this information, the system requires a fee to pay the doctor and manage the medical case. All information sent to the system is coded by using cryptography and only the specialist assigned to the patient will be able to decrypt the data. The patient has control over his medical data. $\mathrm{He} /$ she can easily consult his/her current or previous records, and he/she can partially or completely share it for research activities.

Doctors can register to the system by paying a fee and uploading their credentials such as medical licenses, degrees, and certification documents. Credentials can be verified by any user within the network randomly being invited to become part of a verification committee. Each committee member receives a bonus if he votes to like most of the verifiers. The blockchain system shows the specialist a list of patient cases. Once a case is selected, the specialist can display patient data in order to upload a diagnosis within the system. The doctor receives payment as soon as the patient sends acknowledgment that the data has been received.

The platform also includes a rating system in which doctors are evaluated both by the patient and by the system. In this regard, two different use cases are shown in Figure 4.

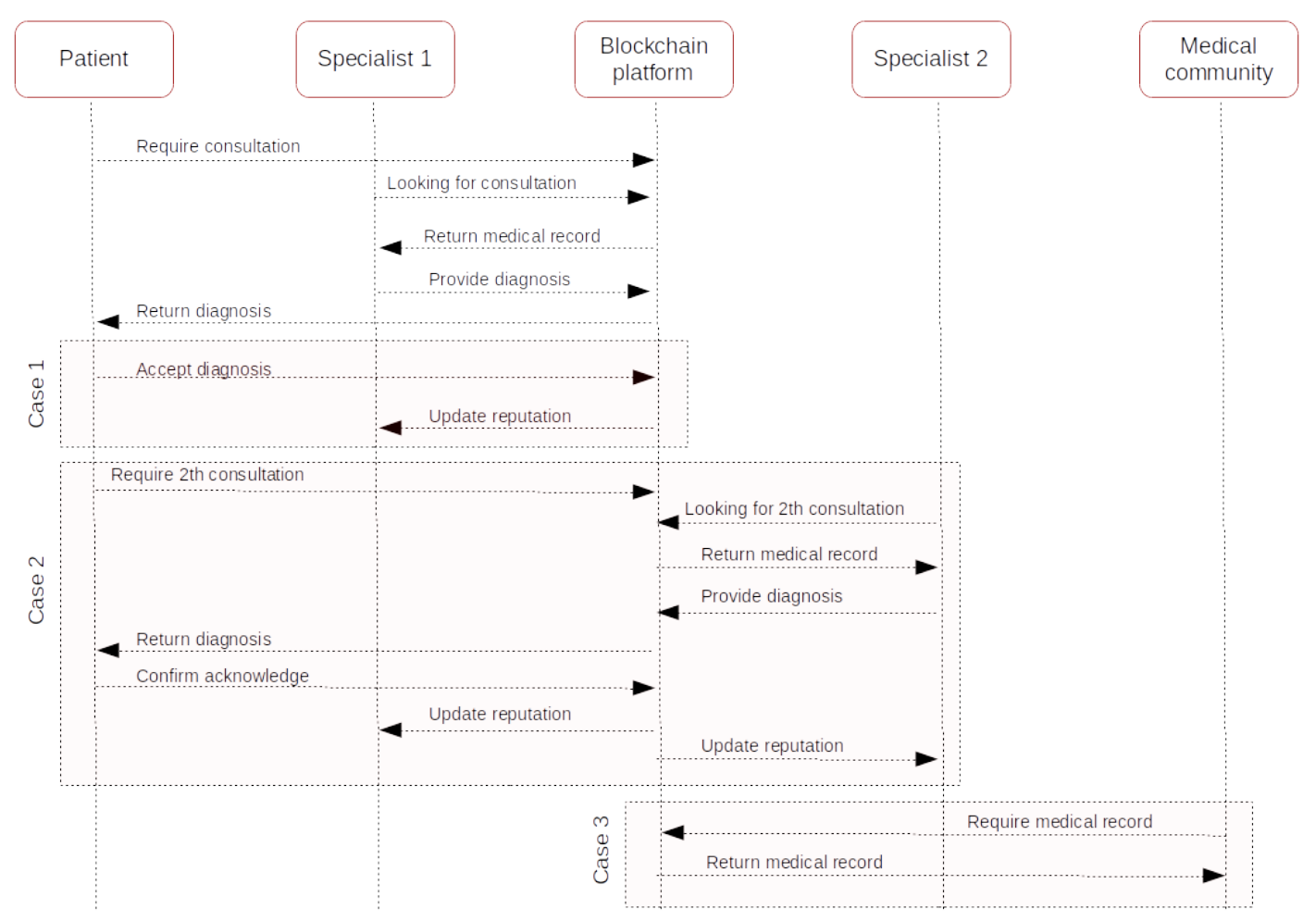

Figure 4. Diagnosis BC procedures: Three use cases.

In the first use case, a patient requires a consultation, and the first specialist (in Figure 4 named "Specialist 1"), provides a diagnosis which can contain recommendations such as a further medical examination. When the patient receives the diagnosis, he/she can accept the response and evaluate the doctor.

In the second use case, the patient may require an additional consultation. In this case, the system requires an extra fee. The reevaluation is assigned to a specialist (in Figure 4 named "Specialist 2"). We assume that the second specialist has better experience and ratings than the first one.

If the second diagnosis is different, the patient is refunded the initial fee and the Specialist 1 does not receive payment. Specialist 2 receives the extra fee and rates Specialist 1 in accordance with their 
diagnosis. If the second diagnosis is the same, Specialist 1 receives the payment and a good rating by the system. Specialist 2 receives the extra fee.

Finally, in a third use case, a medical community member can ask for medical data in order to conduct research activities such as clinical trials or population disease statistics. In this instance, sensitive information is protected. In the case of consent for the processing of sensitive data, information is shared and reachable.

System Architecture

The system can be described as the interaction of four different layers, as shown in Figure 5:

1. Blockchain layer

2. DApp layer

3. API layer

4. Interface layer

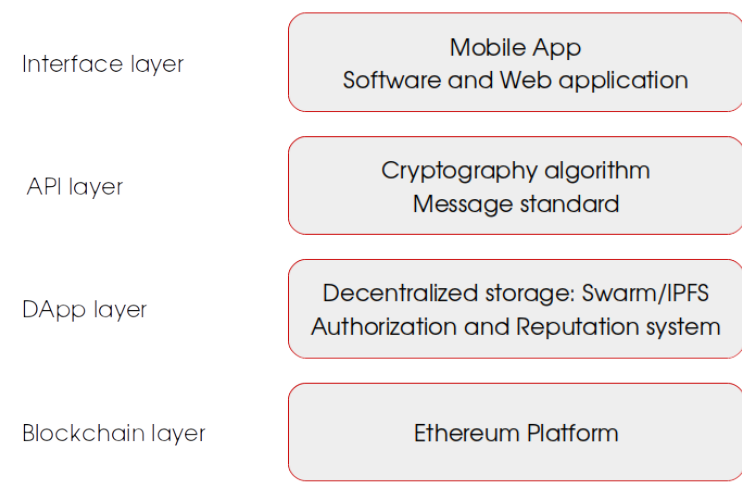

Figure 5. E-health platform structure.

The Blockchain layer represents the lower level of our system. It has the double role of being a communication channel and architectural platform. It is implemented by means of the core technology of the platform, namely the blockchain. As described before, the blockchain provides important characteristics such as immutability and consistency of data, transparency of operations, and authenticity. Because it is based on a peer-to-peer system, it is also fault-tolerant. We chose the Ethereum blockchain because it provides a decentralized platform for applications, in which it is possible to implement our Dapp layer and execute smart contracts. The e-health platform uses the blockchain as communication channel between decentralized applications. Messages between Dapps are structured as Ethereum transactions. Blockchain data is publicly available and in order to protect patients' privacy, each transaction contains requests or data in an encrypted form, according to the API layer specifications.

The DApp layer represents the set of decentralized applications running within the blockchain. Applications in this layer aim to automatically manage and control the whole operation set and the communication system, doing so without intermediaries such as GPs. They take the encrypted messages coming from the API layer and manage them as Ethereum transactions. This layer includes smart contracts that manage transactions within the blockchain, including medical data permissions, and the dermatologists' reputation system. In addition, this layer includes the control of the decentralized database where medical records (including skin photos etc.) are stored. It can be implemented taking into account the Swarm project [31] that aims to provide a new content distribution protocol designed for the Ethereum developer, and simplify content management in a decentralized way. 
The API layer represents protocols and information processing that enable secure and protected information flow between the interface layer and the DApp layer. In particular, it allows the interaction between the user-oriented interfaces and the services in the lower level that become transparent to the users. It includes cryptography protocol that manages and protects data access. Information for each patient request is coded by using symmetric encryption, and therefore the same encryption key is used both to encrypt and decrypt data. In addition, the API layer provides the rules for message composition that will be sent and received by the actors in the system.

The Interface layer represents the higher level of the e-health system and allows user interaction. This layer includes mobile and desktop applications and implements user interfaces for each functionality of the e-health system. As described in Section 3.2, interfaces should be different depending on the actor involved. This is to take in account that patients, doctors, and research community members have different needs. In addition, applications of the interface layer provide functionality of login and registration of users, by means activating the authorization system in the DApp layer. User requests and medical information are transmitted from and to the decentralized system according to the specifications of the API layer.

\section{Discussion}

We designed an e-health blockchain-based platform in addition to that described in Section 3 with the aim of providing the patient with two choices for dermatological consultations.

To sum up, a patient can choose to:

- turn to a general practitioner that logs in the DermoNet platform described in Section 3 which works between the patient and specialist. This can be advantageous for patients that are not familiar with technology, such as elderly people or people that do not have a personal computer, a smartphone, or an Internet connection.

- make use of the blockchain-based platform without a mediator and manage the consultation independently. This second choice has the following advantages, as the patient can:

- be autonomous and control his/her own data;

- reach his medical data from anywhere;

- have a more extensive list of specialists than in the first case.

The DermoNet platform is managed only by healthcare professionals and the patient is supported by medical staff. This solution is particularly suitable in rural area contexts where the ICT infrastructures are poorly developed with respect to health services, or where the low levels of literacy or old age become obstacles. On the contrary, the blockchain e-health platform providing a decentralized system allows for full control of data from patient with the guaranteed of security, transparency and immutability that a centralized system can not ensure. This platform also can provide a global electronic health record system where data will be available for research purposes, teaching, or clinical works. As mentioned above, each actor involved in that system has an account in the Ethereum blockchain which can be identified by an address. The address is unique for each user and each user has his own pair of private/public keys. The login and the use of services are managed by a specific smart contract by according to the user profile.

In the following, we summarize the main advantages of blockchain use.

Innovative medical records. Doctors have patient medical records at their fingertips, and they can keep up to date on the evolution of dermatological diseases. Information on medicines and dates of dermatological visits can be inserted within the blockchain. Every event or transaction, and therefore all information entered, is marked by a date and a timestamp recorded permanently. The management of medical records takes place following an authentication, managed through a smart contract. The information on the patient and therapy is therefore shared securely. 
Improvement of quality. The quality of dermatological care increases. Costs instead decrease thanks to the use of a decentralized system and a distributed peer-to-peer network. The data stored on the blockchain, through the creation of specific smart contracts, can in fact be immediately shared with other doctors and with specific institutions.

Interoperability. Use of the blockchain ensures the security, integrity, and interoperability of healthcare data. Data exchange is cryptographically protected (through Keyless Signature Infrastructure-KSI) with a univocal standard (defined in layer 2). Access to dermatological data is immediate, and the costs of interfacing data from different healthcare systems are eliminated. The data management process is therefore uniform and transparent. The collaboration between patients, researchers, and doctors allows for a greater degree of exchange and comparison, leading to specific and personalized care pathways.

Efficient storage of medical images. Blockchain technology solves problems of dermatological image storage and availability, allowing quick access to information, and timely interpretation of dermatological imaging studies. With the blockchain it is also possible to solve the current problem related to the transfer of image data on printed or optical media. The decentralized diffusion and accessibility of imaging data creates an efficient medical ecosystem and enhances the sharing of information between health organizations.

Transparent management of fiscal aspects (invoicing). Dermatological performance is permanently stored. It is therefore possible to limit health fraud by automating the processing of tickets and invoices and thus reducing administrative costs.

However, there are some cons related to the use of blockchain-based systems:

They represent a new form of technology. The current literature about blockchain is poor. Forums or white papers are currently lacking with respect to the documentation of standards or guidelines to design or implement these kind of systems. No usage feedback or statistical comparisons with traditional systems exists. Furthermore, the lack of standards on integration with other systems results in the need for complete current system redesign.

They are energy-intensive. Each transaction within the blockchain must be verified and validated, requiring high-power computing.

Cultural change. iI is difficult to change people's habits, and in addition, because of the bureaucracy involved, the healthcare industry is not ready for the introduction of such disruptive technology.

\section{Conclusions}

In this paper we presented a blockchain based e-health system with disruptive potential, and compared it to DermoNet, a teledermatology platform implemented using standard technology.

Thanks to the use of cryptography, each participant can decide what information to share, and what to keep confidential.

To summarize, the use of the blockchain proposed system can ensure:

Trust and collaboration. Actors involved rely on technology without knowing each other. Given its decentralized nature, there is no central authority that acts as a guarantor, but it is the entire network that controls management. This type of system promotes cooperation because it leads to an increase in the number of participants that ask for or provide health services in the field of dermatology.

Transparency and security. All transactions are publicly recorded within the blockchain, and no-one can modify records. Although everyone has access to the records, this access is secured through cryptography.

Role identity control and independence. Each user is strongly identified within the system, and depending on their specific profile can access the services. Any participant can interact with the system without a mediator. 
By comparing the two different approaches, we have also focused on the advantages and disadvantages of the two different systems through the underlying main differences and have discussed the limits and applications in different contexts such as rural areas where communication and health services still require development.

The DermoNet model has been designed to become a useful means to speed up medical consultations, to confirm diagnoses, or improve the ability to recognize, diagnose, and treat skin diseases. However, patients do not have direct access to their medical records and they do not manage their data. In addition, the community is limited and supervised by a central authority, subject to Italian law. In reality, the storage and sharing of medical data, while respecting privacy and sensitive information, represent a big challenge. In the future, we plan to design the integration of the two described platforms so that the patient may have the opportunity to receive a more accurate diagnosis, while medical professionals and the research community will have access to a greater range of data and a worldwide health care network.

Acknowledgments: The work presented in this paper was partially funded by Regione Autonoma Sardegna, under a grant for the project Dermonet-P.O.FESR 2007/2013, P.I.A. 2010, (D.G.R. n. 39/3-10/11/2010).

Author Contributions: K.M. contributed to the design and implementation of the Dermonet research project, directed and supervised it. G.B., A.P. and S.I. conceived and designed the proposed blockchain platform. All authors wrote the paper and contributed to the final manuscript.

Conflicts of Interest: The authors declare no conflict of interest.

\section{References}

1. Burdick, A.E.; Berman, B. Teledermatology. Adv. Dermatol. 1997, 12, 19-45.

2. Pak, H.S. Teledermatology and teledermatopathology. Semin. Cutan. Med. Surg. 2002, 21, 179-189.

3. Armstrong, A.W.; Wu, J.; Kovarik, C.L.; Goldyne, M.E.; Oh, D.H.; McKoy, K.C.; Shippy, A.M.; Pak, H.S. State of teledermatology programs in the United States. J. Am. Acad. Dermatol. 2012, 67, 939-944.

4. Mannaro, K.; Baralla, G.; Ibba, S.; Pinna, A.; Garau, C. Towards a smart region: The case study of a teledermatology platform in sardinian region (Italy). In Proceedings of the IEEE 13th International Conference on Wireless and Mobile Computing, Networking and Communications (WiMob), Rome, Italy, 9-11 October 2017; pp. 370-377.

5. Chen, L.; Ho, T.W.; Shih, C.Y.; Lin, F.C.; Lai, F.; Guo, J.W.; Zhuang, M.H. Urban-rural difference in patients utilizing the service of telehealthcare. J. Hosp. Admin. 2015, 4, 56.

6. Khoi, N.M.; Saguna, S.; Mitra, K.; Åhlund, C. Irehmo: An efficient iot-based remote health monitoring system for smart regions. In Proceedings of the 2015 17th International Conference on E-health Networking, Application \& Services (HealthCom), Boston, MA, USA, 14-17 October 2015; pp. 563-568.

7. Khattak, H.A.; Ruta, M.; Di Sciascio, E. CoAP-based healthcare sensor networks: A survey. In Proceedings of the 2014 11th International Bhurban Conference on Applied Sciences and Technology (IBCAST), Islamabad, Pakistan, 14-18 January 2014; pp. 499-503.

8. Aamir, H.; Wenbi, R.; da Silva, A.L.; Nadher, M.; Mudhish, M. Health and emergency-care platform for the elderly and disabled people in the Smart City. J. Syst. Softw. 2015, 110, 253-263.

9. Hossain, M.S. Patient status monitoring for smart home healthcare. In Proceedings of the 2016 IEEE International Conference on Multimedia \& Expo Workshops (ICMEW), Seattle, WA, USA, 11-15 July 2016; pp. 1-6.

10. Kroemer, S.; Frühauf, J.; Campbell, T.M.; Massone, C.; Schwantzer, G.; Soyer, H.P.; Hofmann-Wellenhof, R. Mobile teledermatology for skin tumour screening: Diagnostic accuracy of clinical and dermoscopic image tele-evaluation using cellular phones. Br. J. Dermatol. 2011, 164, 973-979.

11. Rosado, L.; Vasconcelos, M.J.M.; Correia, F.; Costa, N. A novel framework for supervised mobile assessment and risk triage of skin lesions. In Proceedings of the 9th International Conference on Pervasive Computing Technologies for Healthcare, Istanbul, Turkey, 20-23 May 2015; pp. 266-267.

12. Kanthraj, G.R. Patient-assisted teledermatology practice: What is it? When, where, and how it is applied? Indian J. Dermatol. Venereol. Leprol. 2015, 81, 136. 
13. Jaworek-Korjakowska, J.; Tadeusiewicz, R. Design of a teledermatology system to support the consultation of dermoscopic cases using mobile technologies and cloud platform. Bio-Algorithms Med-Syst. 2015, 11, $53-58$.

14. Scheuer, E. Health Information Traceability Foundation. Available online: https://hit.foundation/wpcontent/uploads/2017/12/Whitepaper-HIT-foundation-V1_03.pdf (accessed on 19 February 2017).

15. Petrenko, A.; Kyslyi, R.; Pysmenny, I. Blockchian as a service for medical records. СИСТЕМНI ДОСЛІДЖЕННЯ ТА ІНФОРМАЦЙНІ ТЕХНОЛОГІї 2017, 3, 7-11. (In Russian)

16. Ianculescu, M.; Stanciu, A.; Bica, O.; Neagu, G. Innovative, Adapted Online Services that Can Support the Active, Healthy and Independent Living of Ageing People. A Case Study. Int. J. Econ. Manag. Syst. 2017, 2, 321-329.

17. Shubbar, S. Ultrasound Medical Imaging Systems Using Telemedicine and Blockchain for Remote Monitoring of Responses to Neoadjuvant Chemotherapy in Women's Breast Cancer: Concept and Implementation. Master's Thesis, Kent State University, Kent, OH, USA, 2017.

18. Genestier, P.; Zouarhi, S.; Limeux, P.; Excoffier, D.; Prola, A.; Sandon, S.; Temerson, J.M. Blockchain for Consent Management in the eHealth Environment: A Nugget for Privacy and Security Challenges. J. Int. Soc. Telemed. eHealth 2017, 5, 24.

19. Yue, X.; Wang, H.; Jin, D.; Li, M.; Jiang, W. Healthcare data gateways: Found healthcare intelligence on blockchain with novel privacy risk control. J. Med. Syst. 2016, 40, 218.

20. Lipnack, J.; Stamps, J. Virtual teams: The new way to work. Strategy Leadersh. 1999, 27, 14-19.

21. Staples, D.S.; Hulland, J.S.; Higgins, C.A. A self-efficacy theory explanation for the management of remote workers in virtual organizations. Organ. Sci. 1999, 10, 758-776.

22. Priego-Roche, L.-M.; Verdier, C.; Rieu, D. Virtual Organization Modeling Approach for Home Care Services. In Proceedings of the On the Move to Meaningful Internet Systems: OTM 2013 Workshops, Graz, Austria, 9-13 September 2013; Lecture Notes in Computer Science; Springer: Berlin/Heidelberg, Germany, 2013; Volume 8186, pp. 373-377.

23. Mahmud, H. Modeling Virtual Organization for Home Healthcare Using UML. Int. J. Comput. Sci. Eng. 2016, 4, 22-31.

24. Tamosiunaite, R. Organization virtual or networked? Soc. Technol. 2011, 1, 49-60.

25. Glaser, B.G.; Strauss, A.L. The Discovery of Grounded Theory: Strategies for Qualitative Research; Aldine: New York, NY, USA, 1967.

26. Hennink, M.; Hutter, I.; Bailey, A. Qualitative Research Methods; Sage: London, UK, 2011.

27. Nakamoto, S. Bitcoin: A Peer-to-Peer Electronic Cash System. Available online: https://bitcoin.org/bitcoin. pdf (accessed on 19 February 2018).

28. Buterin, V. Ethereum White Paper. Available online: https://github.com/ethereum/wiki/wiki/White-Paper (accessed on 19 February 2018).

29. Szabo, N. The Idea of Smart Contracts. Available online: http://www.fon.hum.uva.nl/rob/Courses/ InformationInSpeech/CDROM/Literature/LOTwinterschool2006/szabo.best.vwh.net/idea.html (accessed on 19 February 2018).

30. Bartoletti, M.; Pompianu, L. An empirical analysis of smart contracts: Platforms, applications, and design patterns. In Proceedings of the International Conference on Financial Cryptography and Data Security, Sliema, Malta, 7 April 2017; Lecture Notes in Computer Science; Springer: Berlin/Heidelberg, Germany, 2017; Volume 10323, pp. 494-509.

31. Swarm. Available online: https://swarm-guide.readthedocs.io/en/latest/ (accessed on 19 February 2018).

(c) 2018 by the authors. Licensee MDPI, Basel, Switzerland. This article is an open access article distributed under the terms and conditions of the Creative Commons Attribution (CC BY) license (http://creativecommons.org/licenses/by/4.0/). 